\title{
High performance computing aspects of a dimension independent semi-Lagrangian discontinuous Galerkin code $^{\sqrt{2}}$
}

\author{
Lukas Einkemmer ${ }^{\mathrm{a}, *}$ \\ ${ }^{a}$ Department of Mathematics, University of Innsbruck, Austria
}

\begin{abstract}
The recently developed semi-Lagrangian discontinuous Galerkin approach is used to discretize hyperbolic partial differential equations (usually first order equations). Since these methods are conservative, local in space, and able to limit numerical diffusion, they are considered a promising alternative to more traditional semi-Lagrangian schemes (which are usually based on polynomial or spline interpolation).

In this paper, we consider a parallel implementation of a semi-Lagrangian discontinuous Galerkin method for distributed memory systems (so-called clusters). Both strong and weak scaling studies are performed on the Vienna Scientific Cluster 2 (VSC-2). In the case of weak scaling, up to 8192 cores, we observe a parallel efficiency above 0.89 for both two and four dimensional problems. Strong scaling results show good scalability to at least 1024 cores (we consider problems that can be run on a single processor in reasonable time). In addition, we study the scaling of a two dimensional Vlasov-Poisson solver that is implemented using the framework provided. All of the simulation are conducted in the context of worst case communication overhead; i.e., in a setting where the CFL number increases linearly with the problem size.

The framework introduced in this paper facilitates a dimension independent implementation (based on $\mathrm{C}++$ templates) of scientific codes using both an MPI and a hybrid approach to parallelization. We describe the essential ingredients of our implementation.
\end{abstract}

\section{Introduction}

The so-called semi-Lagrangian methods constitute a class of numerical schemes used to discretize hyperbolic partial differential equations (usually first order

\footnotetext{
This work is supported by the Austrian Science Fund (FWF) - project id: P25346. The computational results presented have been achieved using the Vienna Scientific Cluster (VSC).

*Corresponding author

Email address: lukas.einkemmer@uibk.ac.at (Lukas Einkemmer)
} 
equations). The basic idea is to follow the characteristics backward in time. Depending on the equation under consideration, this can be accomplished either by using an analytic formula or an ODE (ordinary differential equations) solver. Note, however, that since the endpoint of a characteristic curve starting at a given grid point usually does not coincide with the grid used, an interpolation procedure has to be employed. An obvious choice is to reconstruct the desired function by polynomial interpolation. However, piecewise polynomial splines are usually preferred.

Note that, such interpolation procedures suffer from a number of shortcomings, two of which we will now discuss in some detail. First, in the case of polynomial interpolations not even conservation of mass is guaranteed (although conservative semi-Lagrangian methods in the context of a finite volume approximation have been considered; see [3]). The traditional approach of dealing with this shortcoming is to employ a high order reconstruction. However, and this brings us to the second shortcoming, such high order reconstructions have to employ large stencils. While the computation of these stencils is usually cheap on a single processor, they incur significant communication overhead when implemented on distributed memory systems. In the case of spline approximations, which according to Sonnendrücker [6] are still considered the de facto standard in Vlasov simulations, a sparse linear system of equations has to be solved (an algorithm with low flop/byte ratio and significant communication overhead).

On the other hand the semi-Lagrangian discontinuous Galerkin methods employ a piecewise polynomial approximation in each cell of the computational domain (see, for example, [11, 4] and [8]). In case of a simple advection equation the represented function is translated and then projected back to the appropriate subspace of piecewise polynomial functions. These methods, per construction, are mass conservative and only access two adjacent cells in order to compute the necessary projection (this is true independent of the order of the approximation). For low CFL numbers (see section 2 for details) this results in a comparable communication overhead as is needed for a low order polynomial interpolation. However, linear and quadratic piecewise polynomials (without a continuity constraint) show a comparable numerical diffusion as a 9 th and 17 th order Lagrange interpolation, respectively. If high frequencies are important, as is usually the case for Vlasov simulations, the piecewise linear interpolation is even comparable to the 17th order Lagrange interpolation (see [4). This can result in a significant reduction in communication overhead (especially for low CFL numbers). Furthermore, the semi-Lagrangian discontinuous Galerkin methods compare very favorable with spline interpolation. However, a direct comparison of the communication overhead in this situation is more difficult as some variant of domain decomposition can be employed in order to improve the scaling of the spline interpolation (see, for example, [2]). Note, however, that even in such a domain decomposition approach a relatively large communication overhead is incurred. This is due to the fact that the boundary condition for the local spline reconstruction requires a large stencil if the desirable properties of the global cubic spline interpolation are to be preserved (the method derived 
in [2] requires a centered stencil of size 21). In addition, the error propagation of the semi-Lagrangian discontinuous Galerkin method, in case of the advection equation, has been investigated and found to be superior to both spline and Lagrange interpolation (see [12] and [9]).

A disadvantage of semi-Lagrangian discontinuous Galerkin methods is that the memory scales linearly in the order of the method. However, since in many applications a relatively low order (compared to other semi-Lagrangian methods) is sufficient and no intermediate coefficients have to be stored in memory (which is the case for the spline interpolation, for example), they constitute a promising numerical method on present and future high performance computing (HPC) systems. Let us note that while well developed numerical software exists for semi-Lagrangian methods employing polynomial and spline interpolation, e.g. the GYSELA code [1] which scales to at least 65000 cores on a Blue Gene/Q system, this is not the case for the discontinuous Galerkin approach considered here

It is therefore our intention to develop a software framework which can be used to implement scientific computer codes based on a semi-Lagrangian discontinuous Galerkin approach. In this paper, we present a dimension independent parallelized implementation of a semi-Lagrangian discontinuous Galerkin discretization (in $\mathrm{C}++$ using MPI and OpenMP). In order to manage code complexity, we will provide a number of dimension independent functions (based on $\mathrm{C}++$ templates) that facilitate the construction of numerical solvers of arbitrary dimension (see section 4). In addition, we analyze weak (problem size is inreased linearly with the number of cores) and strong scaling (problem size is constant as the number of cores increases) behavior for two and four dimensional problems on the VSC-2 HPC system ${ }^{1}$ (see section 3). Furthermore, we briefly discuss a hybrid parallelization strategy (using OpenMP on each socket and MPI between sockets). As an application, we present weak scaling results for a Vlasov-Poisson solver. The Vlasov-Poisson equations are widely used in plasma physics.

\section{The semi-Lagrangian discontinuous Galerkin method}

In this section we describe the semi-Lagrangian discontinuous Galerkin method and its performance characteristics. For simplicity we will limit ourselves to the initial value problem for the advection equation given by

$$
\partial_{t} u(t, x)+v \partial_{x} u(t, x)=0, \quad u(0, x)=u_{0}(x),
$$

where $u$ is the sought after function whereas $v \in \mathbb{R}$ as well as $u_{0}$ are given. For a more detailed treatment we refer the reader to [4] and [5]. The characteristics

\footnotetext{
${ }^{1}$ The VSC-2 (Vienna Scientific Cluster 2) consists of 21024 Opteron Magny Cours 6132HE cores with 2 GB DDR3 memory per core (32 GB per node) and an Infiniband QDR interconnect. See vsc.ac.at for more information. Due to scheduling limitations we have limited our simulations to a maximum of 8192 cores.
} 
of equation (1) can be derived analytically. Therefore, we can write down the exact solution as follows

$$
u(t, x)=u_{0}(x-v t) .
$$

Note that the solution of this problem is the fundamental building block for a splitting approach to the Vlasov-Poisson and Vlasov-Maxwell equations. It can also be used to handle a Burgers' type nonlinearity (see [7]).

Now, let us consider the discretization of this equation within the semiLagrangian discontinuous Galerkin approach. We divide our domain in cells $C_{i}$ and assume that a function $\tilde{u}$ is given such that $\left.\tilde{u}\right|_{C_{i}}$, i.e. the restriction of $\tilde{u}$ to the $i$ th cell, is a polynomial of degree $k$. Then the function $\tilde{u}$ lies in the approximation space. However, the translated function $x \mapsto \tilde{u}(x-v \tau)$, where $\tau$ is the time step size, can, in general, not be represented in this form. Therefore, we use a projection operator $P$ and compute (numerically)

$$
\tilde{u}^{n+1}=P \tilde{u}^{n}(\cdot-v t) .
$$

The function $\tilde{u}^{n+1}$ constitutes the sought after approximation of $\tilde{u}(x-v \tau)$. The operator $P$ is the $L^{2}$ projection on the (finite dimensional) subspace of cell-wise polynomials of degree $k$.

To determine the numerical method we have to chose a basis of the finite dimensional subspace. This then also determines the degrees of freedom stored in computer memory. In [8] the basis of Legendre polynomials up to degree $k$ has been employed. The projection can then be computed either analytically or by Gaussian quadrature. On the other hand in 4 the degrees of freedom are chosen as the Gauss-Legendre points in each cell (this corresponds to a basis of certain Lagrange basis polynomials). The projection is then computed by Gaussian quadrature. The numerical effort of both implementations is comparable. In the implementation considered here we employ the latter approach. This is due to the fact that the degrees of freedom correspond to function evaluations at given points. This makes the extension to arbitrary dimensions easier and facilitates the interoperability with third party libraries. The explicit formulas necessary for the implementation are derived and stated in 4 .

To conclude this section, let us discuss the computational effort and communication overhead of the semi-Lagrangian discontinuous Galerkin method considered here. Counting the number of arithmetic operations for every of the $n^{d} o^{d}$ degrees of freedom, where $n$ is the number of cells, $o=k+1$ is the order of the polynomial approximation, and $d$ is the space dimension, we obtain a total of $16 o^{3}+15 o^{2}+7 o+3$ floating point operations per time step. For piecewise constant, linear, and quadratic polynomials the flops per step and the flops per byte (read from main memory) are shown in Table 1 .

Let us note that the problem under consideration is compute bound on most modern architectures for polynomials of degree one or above. The implementation is currently not aggressively optimized, in order to maintain code readability, and no effort has been made to vectorize the code (besides the vectorization that is performed by the compiler). 


\begin{tabular}{ccc}
\hline$o$ & flops/step & flops/byte \\
\hline 1 & $41 n^{d} o^{d}$ & 2.6 \\
2 & $205 n^{d} o^{d}$ & 12.8 \\
3 & $591 n^{d} o^{d}$ & 36.9
\end{tabular}

Table 1: Number of floating point operation per time step are shown for the semi-Lagrangian discontinuous Galerkin scheme considered in this paper. In addition, the flop to byte ratio for double precision floating point numbers is displayed in terms of the number of cells $n$ and the order of the approximation in each cell $o$. If single precision arithmetics is used, the flop/byte ratio is halved.

Let us now discuss the communication overhead necessary on a distributed memory model. The scheme under consideration, as is true for semi-Lagrangian methods in general, is not restricted by a CFL (Courant-Friedrichs-Lewy) condition. In what follows we denote the CFL number ${ }^{2}$ by $C$. Each MPI process communicates $n_{0}^{d-1}\lceil C\rceil o$ double precision floating points numbers to exactly one adjacent MPI process, where $n_{0}^{d}$ is the number of floating point numbers stored per process.

\section{Numerical results}

In this section we provide weak and strong scaling results for the advection problem introduced in section 2. As the metric of merit we use parallel efficiency, which is defined as the (theoretical) run time of a program that scales ideally divided by the measured run time. In addition, we discuss an implementation of a Vlasov-Poisson solver (based on the discontinuous Galerkin semiLagrangian framework discussed here). Before proceeding, let us note that the communication overhead of our computer program is due to MPI communication, preparation of the boundary data necessary for this communication, and synchronization overhead. It is essential for an efficient implementation that the MPI communication is interleaved with computation (to hide the associated communication overhead). However, even if this is accomplished, as is the case for most weak scaling studies conducted in this paper, the parallel efficiency is influenced by the two other factors as well.

First, let us conduct a weak scaling study of the two dimensional advection equation. Since for a two dimensional problem we can run numerical simulations of appreciable size (at least up to 1024 cells in each direction) on a single node, we will consider both weak and strong scaling here. In all the simulations conducted we fix the time step size. Therefore, the CFL number, and thus the amount of data communicated by any two processors increases linearly with the problem size. Let us note that this configuration constitutes the worst case as far as communication overhead is concerned. In a realistic simulation, however, we would most likely have to decrease the time step size simultaneously with

\footnotetext{
${ }^{2}$ In case of the advection equation considered in this section, the CFL number is defined as $\tau v$ divided by the cell size, where $\tau$ is the time step size and $v$ the advection speed.
} 


\begin{tabular}{lrr}
\hline$\#$ cores & time & efficiency \\
\hline 16 & 14.36 & 1.00 \\
64 & 14.80 & 0.97 \\
256 & 14.88 & 0.96 \\
1024 & 15.28 & 0.94 \\
2048 & 15.23 & 0.94 \\
4096 & 15.26 & 0.94
\end{tabular}

\begin{tabular}{lrr}
\hline \# cores & time & efficiency \\
\hline 16 & 14.90 & 1.00 \\
64 & 15.34 & 0.97 \\
256 & 15.03 & 0.99 \\
1024 & 15.37 & 0.97 \\
2048 & 15.71 & 0.95 \\
4096 & 15.58 & 0.96 \\
8192 & 16.09 & 0.93
\end{tabular}

Table 2: Weak scaling for the two dimensional advection equation using $512 \times 512$ cells (left) and $1024 \times 1024$ cells (right) with piecewise polynomials of degree $1(o=2)$ per MPI process. The run time as well as the parallel efficiency, compared to a single node, is shown (for ideal scaling the measured run time would remain constant). The CFL number for $512 \times 512 / 1024 \times 1024$ cells per MPI process ranges from approximately $0.5 / 1$ for a single core to approximately $32 / 91$ for $4096 / 8192$ cores.

\begin{tabular}{lrr}
\hline \# cores & time & efficiency \\
\hline 16 & 59.49 & 1.00 \\
64 & 16.47 & 0.90 \\
256 & 4.71 & 0.79 \\
1024 & 1.80 & 0.52
\end{tabular}

\begin{tabular}{lrr}
\hline \# cores & time & efficiency \\
\hline 16 & 51.47 & 1.00 \\
64 & 12.93 & 1.00 \\
256 & 3.40 & 0.95 \\
1024 & 0.98 & 0.82 \\
2048 & 0.55 & 0.73
\end{tabular}

Table 3: Strong scaling for the two dimensional advection equation using a total of $256 \times 256$ cells with a CFL number of approximately 1 (left) and $1024 \times 1024$ cells with a CLF number of approximately 5 (right). In both problems piecewise polynomials of degree $1(o=2)$ are employed. The run time as well as the parallel efficiency is shown (for ideal scaling the measured run time would decrease linearly with the number of cores used).

increasing the problem size in order to keep the time integration error sufficiently small.

The weak scaling results are given in Table 2. We observe excellent scaling for up to 8192 cores. Furthermore, the timing results provided by the computer program do suggest that we are able to hide the MPI communication overhead almost perfectly up to the maximum number of cores considered here. Thus, we would expect that this implementation scales to larger HPC systems as well.

Now, let us consider the strong scaling results given in Table 3. In this case the communication overhead increases with the number of cores while the computation time decreases. Therefore, starting at a certain number of cores (depending on the problem size) we are not able to hide the communication overhead anymore and the parallel efficiency degrades significantly. Let us further note that the choice of the CFL number has a significant influence on the strong scaling that is achieved in such simulations. This is due to the fact that the communication overhead increases linearly with the CFL number.

Second, let us consider the four dimensional advection equation. In this case we can not run a problem of appreciable size on a single core. Thus, we will only consider weak scaling here. The corresponding results are given in 


\begin{tabular}{|c|c|c|c|c|c|}
\hline$\#$ cores & time & efficiency & \# cores & time & efficiency \\
\hline 16 & 6.00 & 1.00 & 16 & 12.50 & 1.00 \\
\hline 256 & 6.23 & 0.96 & 256 & 13.78 & 0.91 \\
\hline 1024 & 6.32 & 0.95 & 1024 & 13.89 & 0.90 \\
\hline 2048 & 6.37 & 0.94 & 2048 & 15.12 & 0.83 \\
\hline 4096 & 6.71 & 0.89 & 4096 & 15.65 & 0.80 \\
\hline 8192 & 6.89 & 0.87 & & & \\
\hline
\end{tabular}

Table 4: Weak scaling for the four dimensional advection equation using $16^{4}$ cells (left) and $32^{4}$ cells (right) with piecewise polynomials of degree $1(o=2)$ per MPI process. The run time as well as the parallel efficiency, compared to a single node, is shown (for ideal scaling the measured run time would remain constant). For $16^{4} / 32^{4}$ cells per MPI process the CFL number ranges from approximately $0.6 / 1.3$ for a single core to approximately $6 / 10$ for $8192 / 4096$ cores.

Table 4. Let us make two observations. First, while we observe good scaling up to 8192 cores, the parallel efficiency is lower than in the case of the two dimensional advection. This, however, is expected as the ratio of computation to communication overhead is significantly lower in the four dimensional problem (as compared to the two dimensional problem considered above).

Third, let us consider the Vlasov-Poisson equations as an example of an application of the semi-Lagrangian discontinuous Galerkin framework discussed in this paper. We employ the numerical approach outlined in [8] and use the FFTW [10] library to compute a solution to Poisson's equation. Once again, we employ a fixed time step size which results in a CFL number that scales linearly with the problem size. The weak scaling results for this configuration are shown in Figure 1. In the plot on the right we show the run time of the different parts of the program as a function of the number of cores used. Note that while the computational effort of Poisson's equation is negligible (as is widely recognized in the literature as Poisson's equation is posed in a single space dimension only), the communication time necessary for the three advections is similar to that of the fast Fourier transform. This additional communication overhead, due to the collective communication necessary to solve Poisson's equations, accounts for the slight additional decrease in parallel efficiency as compared to the advection problem considered in Table 2, Furthermore, let us note that a more detailed analysis reveals that the communication for the three advections is almost completely hidden by the corresponding computation. The measured time (as shown in the plot) is mainly due to the preparation of the boundary data (i.e., populating the proper data structure) and synchronization overhead.

To complete this section, let us briefly discuss hybrid programming. All the simulations above are conducted using MPI for parallelization. A hybrid approach complements this by using OpenMP on each node and MPI to communicate between different nodes. Such a hybrid implementation has the (potential) advantage that less communication overhead is incurred (each node represents a shared memory architecture). We have implemented this hybrid programming model for the simulations conducted above. However, up to the number 


\begin{tabular}{lrr}
\hline$\#$ cores & time & scaling \\
\hline 16 & 21.18 & 1.00 \\
64 & 22.43 & 0.94 \\
256 & 23.70 & 0.89 \\
1024 & 24.03 & 0.88 \\
2048 & 24.31 & 0.87 \\
4096 & 24.76 & 0.86 \\
8192 & 24.30 & 0.87
\end{tabular}

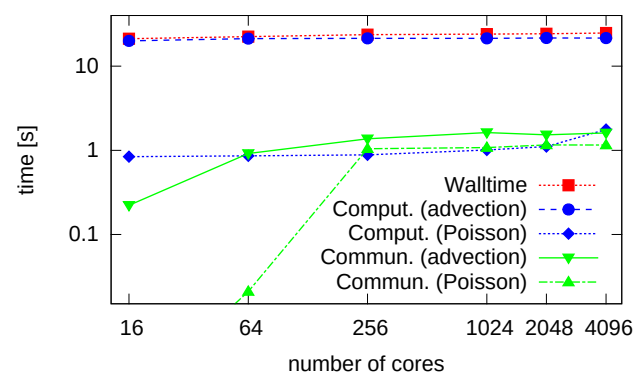

Figure 1: Weak scaling for the two dimensional Vlasov-Poisson equation using $512 \times 512$ cells $(n=512)$ and piecewise polynomials of degree $1(o=2)$ per MPI process. The run time as well as the scaling behavior is shown (for ideal scaling the measured run time would remain constant). The time step size is chosen as $\tau=0.01$. This results in a worst case CFL number of approximately 2.5 for a single core and approximately 221 for 8192 cores. The plot on the right shows the run time (of the different parts of the program) as a function of the number of cores.

of cores considered here, we have not observed a significant increase in performance compared to the MPI only implementation. Let us note, however, that in case of simulations which have a very large worst case CFL number (such as for initial values of the Vlasov-Poisson equations that decay only algebraically) it enables us to consider larger problem sizes while still maintaining a communication scheme where each MPI process exclusively talks with its neighbors. In addition, a slight decrease in the total amount of memory consumed can be observed for the hybrid implementation (up to approximately 15\%).

\section{Description of the computer code}

In this section we will describe the three main aspects of the implementation necessary to extend existing codes (such as the advection and Vlasov-Poisson implementation used in the previous section) and to implement new schemes based on the semi-Lagrangian discontinuous Galerkin framework described here.

The dimension of the problem is a template parameter denoted by d. Thus, in order to change the dimension of a given implementation we have to recompile the application. This, in our view, is not a serious limitation; furthermore, it enables the compiler to apply additional optimizations for each use case. Also, in this framework it is very simple to supply specific optimizations which can only be applied to a two dimensional problem, for example. As the index type we use the typesafe boost: : array class from the Boost library.

In developing dimension independent scientific codes it is vital to have a construct that enables us to loop over all (or a subset) of the indices in a multi-dimensional array. Our framework provides the function iter_next to facilitate this behavior. Its prototype and a usage example are given in the following listing. 


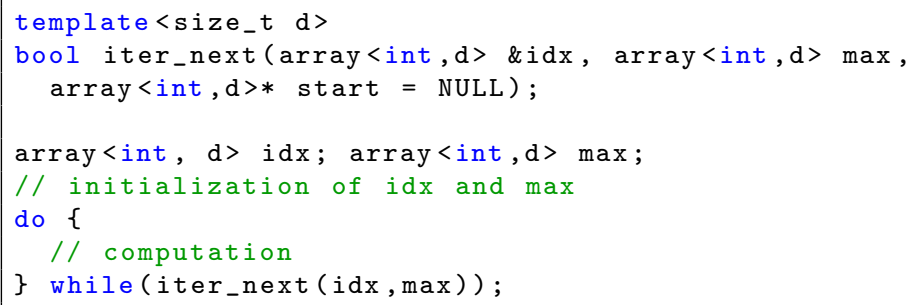

The argument idx specifies the current (multi-)index and max denotes the upper bound of the iteration in each direction. In addition, the optional third parameter can be used to specify a starting value that is different from zero in one or more directions.

In order to perform communicate over the MPI interface the appropriate slice of the multi-dimensional data has to be extracted. To facilitate this, in a dimension independent way, the following two functions are provided.

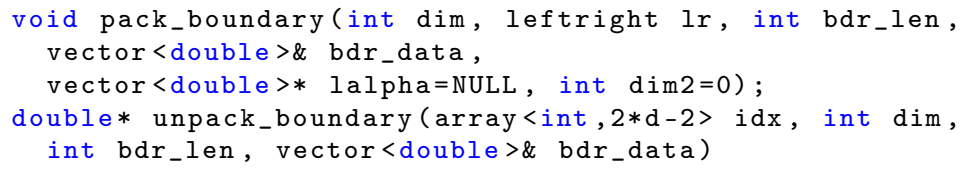

The user has to specify the dimension in which the translation is to be conducted (dim), the number of cells that need to be communicated (in the dim-direction), and a vector to hold the boundary data (bdr_data). In addition, the direction of the translation is given ( $l r)$; alternatively, also a vector of advection speeds can be specified (lalpha). In the latter case the direction of the translation can be different at each degree of freedom (in the dim2-direction). The vector bdr_data is then send via an appropriate MPI call. The receiving process uses the unpack_boundary function in order to determine the appropriate pointer that is passed to the translate1d function.

The translate1d function performs the actual computation of the advection and its prototype is given in the following listing.

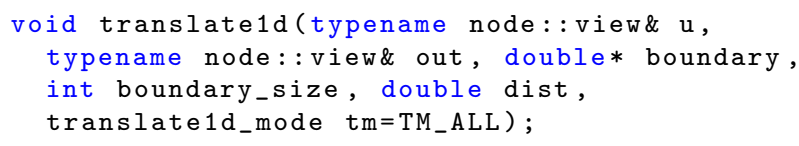

The first two arguments specify the input and output iterator, respectively. The pointer to the appropriate boundary data is determined by the unpack_boundary function. In addition, we have to specify the number of cells in the translation direction (boundary_size), and the distance (dist) of the translation. In addition, for the last argument we can specify TM_INTERIOR. In this case only the part of the computation that can be done without knowing boundary is performed (in this situation it is permissible to pass a NULL pointer as the third argument). This then has to be followed up, in order to complete the computation, by an additional call which specifies TM_BOUNDARY as the last argument 
and a proper pointer to boundary data. This is useful for interleaving communication with computation and thus has been applied in all the numerical simulations conducted in section 3 .

The source code can be build using the included Makefile; it generates an executable named sldg that can be executed as follows

./sldg identifier

where identifier is one of test_1d_order, test_2d_order (checks the order of the approximation for a simple advection), test_1d_mpi, test_2d_mpi, test_2d_hybrid, test_4d_mpi, test_4d_hybrid (solves the advection equation in parallel), and vlasovpoisson (solves the Vlasov-Poisson equations in parallel). Further options can be specified. A overview of available options is displayed by running

. / sldg - - help

The current version of the software can be obtained from https://bitbucket. org/leinkemmer/sldg.

\section{Conclusion \& Outlook}

The results presented in this paper show that the semi-Lagrangian discontinuous Galerkin approach can be parallelized efficiently to at least 8192 cores on a modern HPC system. It is our believe that the framework introduced here greatly facilitates the implementation of scientific computer codes based on this discretization. As future work we will consider two extensions of the implementation presented here.

First, many modern HPC systems include up to a few hundred thousand cores. Therefore, to investigate and optimize scaling to larger HPC systems, compared to what we have considered in this paper, is certainly of interest. This would also allow us to treat five or even six dimensional problems (which is of interest in the study of kinetic plasma models, for example). In addition, improving the single threaded performance by vectorization would conceivably lead to significant gains in performance.

Second, in this paper we have only considered briefly the application to problems of practical interest. In this context, we are mostly interested in kinetic models of plasma physics (such as the Vlasov-Poisson equations, VlasovMaxwell equations, and gyrokinetic models) and in advection dominated second order partial differential equations. In the latter case a splitting approach has to be employed, which allows us to treat the diffusion term separately.

\section{References}

[1] J. Bigot, V. Grandgirard, G. Latu, C. Passeron, F. Rozar, and O. Thomine. Scaling GYSELA code beyond 32K-cores on Blue Gene/Q. In ESAIM: Proceedings, volume 43, pages 117-135, 2013. 
[2] N. Crouseilles, G. Latu, and E. Sonnendrücker. A parallel Vlasov solver based on local cubic spline interpolation on patches. J. Comput. Phys., 228(5):1429-1446, 2009.

[3] N. Crouseilles, M. Mehrenberger, and E. Sonnendrücker. Conservative semi-Lagrangian schemes for Vlasov equations. J. Comput. Phys., 229(6):1927-1953, 2010.

[4] N. Crouseilles, M. Mehrenberger, and F. Vecil. Discontinuous Galerkin semi-Lagrangian method for Vlasov-Poisson. In ESAIM: Proceedings, volume 32, pages 211-230. EDP Sciences, 2011.

[5] N. Crouseilles, M. Mehrenberger, and F. Vecil. A discontinuous Galerkin semi-Lagrangian solver for the guiding-center problem. HAL preprint, hal$00717155,2012$.

[6] E. Sonnendrücker. Numerical methods for the Vlasov equation. http://icerm.brown.edu/html/programs/sp_f11/schedules/ slides_workshop_1_tutorial/LectureNumericalVlasov.pdf, 2011.

[7] L. Einkemmer and A. Ostermann. A splitting approach for the KadomtsevPetviashvili equation. arXiv preprint, arXiv:1407.8154, 2014.

[8] L. Einkemmer and A. Ostermann. Convergence analysis of a discontinuous Galerkin/Strang splitting approximation for the Vlasov-Poisson equations. SIAM J. Numer. Anal., 52(2):757-778, 2014.

[9] L. Einkemmer and A. Ostermann. On the error propagation of semiLagrange and Fourier methods for advection problems. To appear in Comput. Math. Appl., 2014.

[10] M. Frigo and S.G. Johnson. The Design and Implementation of FFTW3. Proceedings of the IEEE, 93(2):216-231, 2005. Special issue on "Program Generation, Optimization, and Platform Adaptation".

[11] J.M. Qiu and C.W. Shu. Positivity preserving semi-Lagrangian discontinuous Galerkin formulation: theoretical analysis and application to the Vlasov-Poisson system. J. Comput. Phys., 230(23):8386-8409, 2011.

[12] C. Steiner, M. Mehrenberger, and D. Bouche. A semi-Lagrangian discontinuous Galerkin superconvergence. HAL preprint, hal-00852411, 2013. 